\title{
Minute Times Microgram Per Milliliter Per Gram
}

National Cancer Institute

\section{Source}

National Cancer Institute. Minute Times Microgram Per Milliliter Per Gram. NCI

Thesaurus. Code C112338.

Minutes times micrograms per milliliter, divided by grams. 